\title{
Oil-gas-water three-phase upward flow through a vertical pipe: Influence of gas injection on the pressure gradient
}

\author{
Jing-yu Xu* , Jian Zhang, Hai-fei Liu, Ying-xiang Wu \\ LHO, Institute of Mechanics, Chinese Academy of Sciences, Beijing 100190, China
}

\section{A R T I C L E I N F O}

\section{Article history:}

Received 23 December 2011

Received in revised form 31 March 2012

Accepted 5 June 2012

Available online 15 June 2012

\section{Keywords:}

Oil-gas-water flow

Pressure gradient

Phase inversion

Vertical pipe

\begin{abstract}
A B S T R A C T
An experimental study of three-phase flow in a vertical pipe with $50 \mathrm{~mm}$ diameter has been carried out. The experiments were conducted under the input superficial phase velocity: water from 0 to $0.885 \mathrm{~m} / \mathrm{s}$, oil from 0 to $0.90 \mathrm{~m} / \mathrm{s}$ and gas from 0 to $0.85 \mathrm{~m} / \mathrm{s}$. In order to investigate the influence of gas injection on an oil-water two-phase flow, the average in situ phase fraction and pressure gradient were measured. The results showed that gas injection had little impact on the phase invasion and the phase inversion was still taking place at the same oil volume fraction. The average in situ gas fraction reached to its lowest value around the phase inversion point. In comparison to oil-water flow, the presence of gas decreased considerably the gravity pressure gradient and the total pressure gradient was reduced. However, when the input oil fraction was close to the phase inversion point, the contribution of frictional and gravity terms to the total pressure gradient became more equal, hence the total pressure gradient in three-phase flow could be higher than that in oil-water flow around the phase inversion region. In addition, several methods based on an oil-water flow as a single phase flow were evaluated to predict the pressure gradient of three-phase vertical bubbly flow. A good agreement was obtained between theory and data in this work and those in the literature.
\end{abstract}

(ㄷ) 2012 Elsevier Ltd. All rights reserved.

\section{Introduction}

In the petroleum industry, oil-gas-water three-phase vertical flow often occurs. For instance, the gas-lift technique is often applied in oil well. This gravity-driven pumping technique enhances oil production by injecting gas at the bottom of a production pipe, which can reduce the bottom-hole pressure and lead to more inflow of oil into the well bore. In recent years, because of the presence of water in oil well or injection of water into the well for increasing oil production, oil extraction is often accompanied by a high water throughput. Therefore, oil production results in transportation of oil-gas-water three-phase flow in vertical pipes when the gas-lift technique is also applied. There are many articles dealing with three-phase flow through a vertical pipe (Woods et al., 1998; Spedding et al., 2000; Bannwart et al., 2009; Cazarez et al., 2010). However, not much research has been done concerning the effect of gas injection on pressure gradient in an oil-water vertical flow (Descamps et al., 2006, 2007). In the works of Descamps et al. an experimental investigation was made of three-phase flow in a vertical pipe. It was shown that with air injection the pressure gradient of three-phase flow was always significantly smaller than for the case of oil-water flow, except at the point of phase inversion where

\footnotetext{
* Corresponding author. Tel.: +86 108254 4179; fax: +86 1062561284 .

E-mail address: xujingyu@imech.ac.cn (J.-y. Xu).
}

the pressure gradient could be even be higher than for oil-water flow. In order to extend the knowledge of the effect of gas injection on the flow characteristics of immiscible liquids, we have made an experimental investigation of an oil-gas-water flow through a vertical pipe. Special attention focuses on influence of gas injection on the pressure gradient. To calculate the friction factors the average in situ fractions of three-phase are measured, respectively, according to the slip among three phases. The experimental results of three-phase flow, for the phase inversion and the pressure gradient, are compared with those measured in the oil and water two-phase flow (Xu et al., 2010). In addition, a simple relationship for calculating the law for wall shear stress ratio is developed for an oil-gas-water three-phase vertical flow.

As a reference we start with the results about the pressure gradient without gas injection. Thereafter, the influence of gas injection on the pressure gradient will be presented. Finally, several methods are evaluated to predict the pressure gradient of threephase vertical bubbly flow.

\section{Experimental facility and measurement procedure}

An experimental facility was fixed to simulate flow conditions in vertical pipes and be able to identify and characterize the flow patterns and measure the pressure gradient and average in situ phase fraction. A schematic diagram of the experimental system 
was shown in Fig. 1. The two immiscible liquids used were tap water and white oil (density $860 \mathrm{~kg} / \mathrm{m}^{3}$, viscosity $0.044 \mathrm{~Pa} \mathrm{~s}$ at $20{ }^{\circ} \mathrm{C}$ and oil-water interracial tension $0.031 \mathrm{~N} / \mathrm{m}$ ). Air was used as gas phase (air-water surface tension $0.071 \mathrm{~N} / \mathrm{m}$, air-oil surface tension $0.044 \mathrm{~N} / \mathrm{m}$ ). A transparent perspex pipe with an inner diameter of $50 \mathrm{~mm}$ was used. Prior to the vertical pipe, white oil and water were pumped from their respective storage tanks, metered, and introduced into a horizontal section of 140 internal diameters via a T-junction, which ensured minimum mixing. And then, after a vertical upward development section of 40 internal diameters, the mixture fluids flowed through the test section. When the steady state of oil and water flow was reached under a flow condition, injection of gas was started. Based on the experimental observation, the length of horizontal piece had few influences on the flow pattern for the vertical test section. In fact, the length of the vertical development section had certain influences on the flow structure. In this experiment, we set a vertical section of 40 internal diameters to stabilize the flow. Due to the fact that the mixture velocity of three-phase was low (Um $<3 \mathrm{~m} / \mathrm{s}$ ) and oil phase viscosity high ( $44 \mathrm{mPa} \mathrm{s})$, this length provided sufficient length to stabilize the flow. Furthermore, air was stored in a gas bottle (pressurized at 10 bar with a reduction to 3 bar) and monitored by a rotameter. The nozzle, which was a small tube with an internal diameter of $3.5 \mathrm{~mm}$ located in the bottom of the vertical pipe generating bubbles, was used as an injector.

The research was restricted to three-phase bubbly or intermittent flow with a continuous liquid (oil or water), in which the other liquid and gas were present. Experiments were carried out either by keeping the mixture liquids velocity constant and increasing the gas velocity or by keeping the superficial water/gas velocity constant and increasing the superficial oil velocity. Measurements were made for input water velocity from 0 to $0.885 \mathrm{~m} / \mathrm{s}$, input oil velocity from 0 to $0.90 \mathrm{~m} / \mathrm{s}$ and input gas velocity from 0 to $0.85 \mathrm{~m} / \mathrm{s}$. During the experiment the temperature with a thermocouple was mounted in the pipe wall.

Pressure gradient in the test section was measured by two absolute pressure transducers and a differential pressure transducer. Accurate results of total pressure gradient could obtained from the difference of the measurements between the two transducers which were located at the $1.0 \mathrm{~m}$ long test section. The sampling frequency of the pressure was $500 \mathrm{~Hz}$ and a total of 60,000 samples, which corresponded to two minutes sampling time, were averaged. Most of the experiments were conducted more than at least two times and the reproducibility of the experiments was good.

The average in situ phase fraction was obtained by the rapid closing valve method. It included two rapid closing valves installed on run tube with $50 \mathrm{~mm}$ diameter, and two rapid closing valves on the bypass tube with $25 \mathrm{~mm}$ diameter. The rapid closing valves connected by mechanical linkages were installed in the test section at a distance of $1.5 \mathrm{~m}$. The bypass was implemented to switch the flow between the run and the bypass when the two valves on run were closed to measure the average phase holdup. During the measurements runs, sufficient time was taken to allow fully developed flow to be established. The rapid closing valves were then closed to get a representative sample of two- or three-phase flows for phase volume fraction measurement. The sample was transferred to a graduated cylinder by carefully purging the liquid phases using pressurized gas in order to assure not to leave any liquid inside of the test section. The samples of the liquid phases were left in graduated cylinders for a time period of about $6 \mathrm{~h}$ to assure virtually complete gravitational phase separation before measurements of the liquid phase volumes were done. By taking repeated samples it was found that the fluctuation of mean value of the measurements over the three times was around $7.6 \%$. Finally, the average in situ phase fractions were calculated using the each phase volumes.

\section{Oil-water flows without gas injection}

In the previous experimental studies (Xu et al., 2010), many phase inversion and pressure gradient experiments were performed in an oil-water upward flow through a vertical pipe. In this section the results are briefly summarized to help in the interpretation of the experiments with gas injection, which will be presented in the coming sections. In the earlier work the flow pattern maps was observed and the identity of the continuous phase was made. The flow pattern maps showing the superficial velocities are demonstrated in Fig. 2. The solid line represents

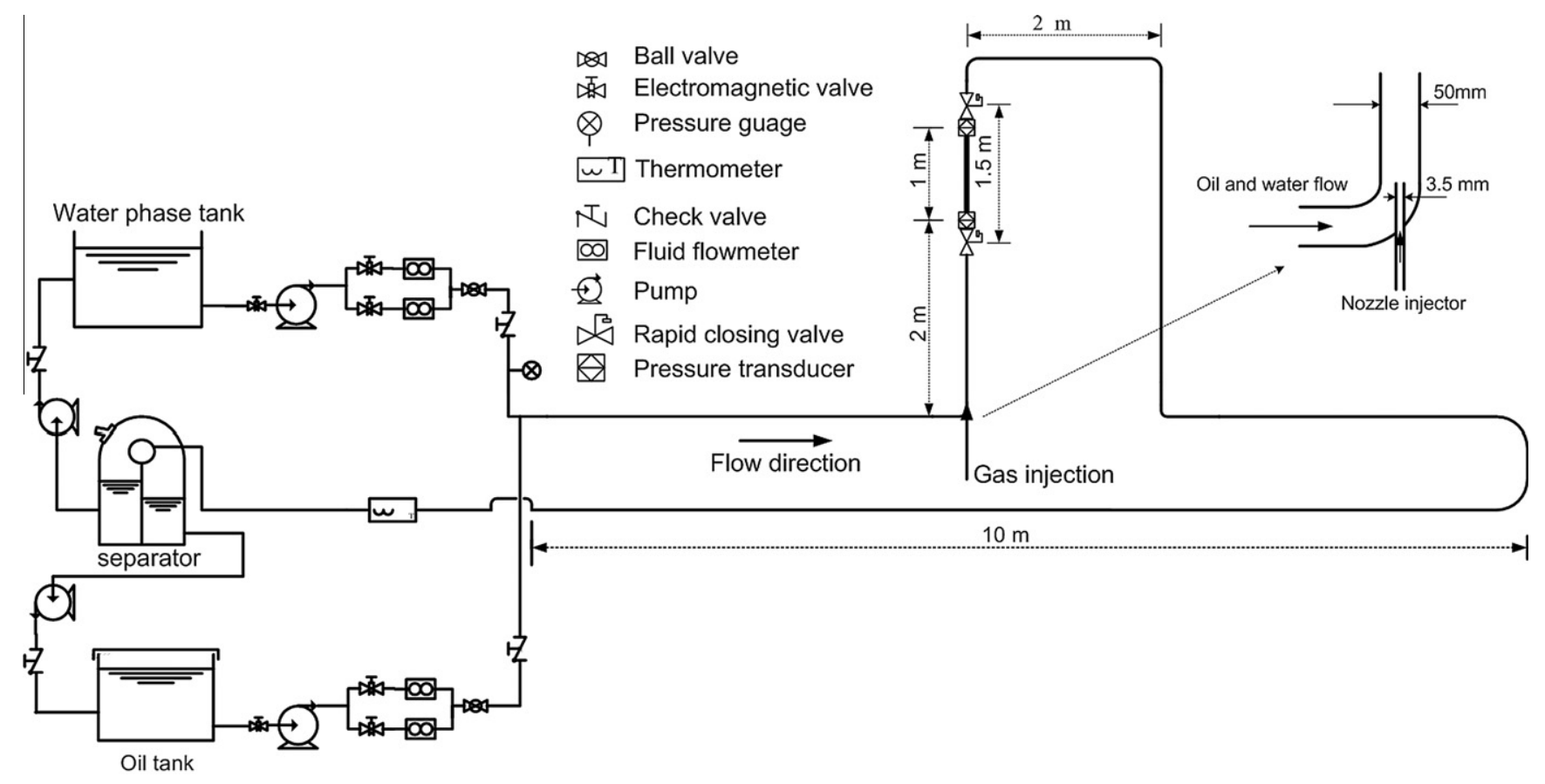

Fig. 1. Schematic view of the flow loop. 


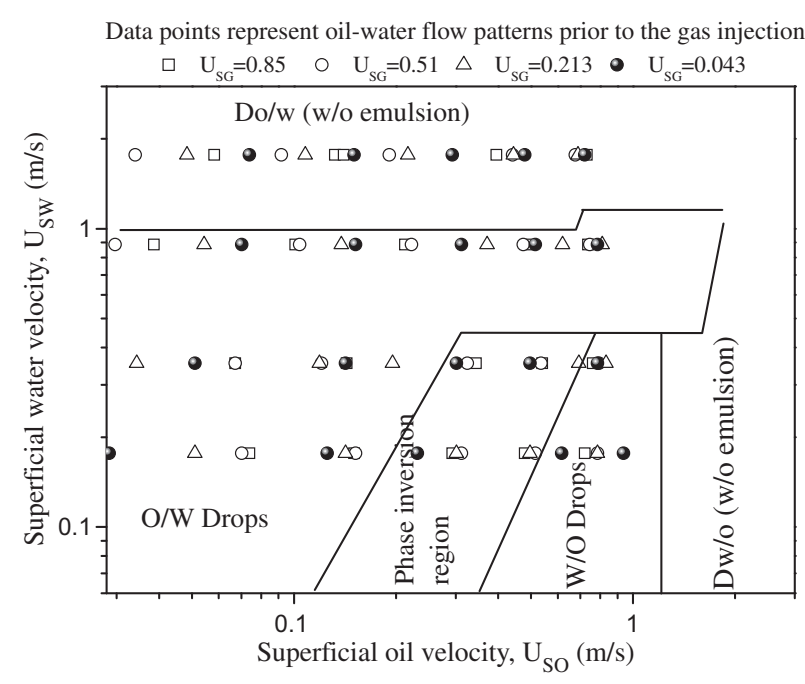

Fig. 2. Flow pattern map of oil-water upward flow in a vertical pipe.

the boundary of the experimental data. As be shown in the figure, the region of the water-dominated flow is larger than that of the oil-dominated flow. During these experiments it is noticed that when an experiment is carried out by keeping superficial water velocity constant and increasing the dispersed oil phase velocity, the flow structures can change from o/w drops flow to the complex structure flow (phase inversion region), and then to w/o drops flow. The phase inversion has become of importance in the long pipeline transportation of oils, due to the fact that there is an abrupt and significant change in the frictional pressure gradient associated in the region, where phase inversion from water to oil continuous occurs. Based on the experimental results of oil-water vertical flow, the phase inversion takes place at an input oil fraction of about $80 \%$ regardless of the mixture velocity.

In Fig. 3, the total pressure gradient, and its two component parts of gravity and frictional pressure gradients, measured for an oil-water flow is presented as a function of the mixture liquid velocity for three different values of the superficial water velocity. As can be seen the total pressure gradient gradually decreases and reaches a minimum with increasing the mixture liquids velocity. Thereafter, the total pressure gradient increases with the velocity further increased. This phenomenon is observed obviously for a

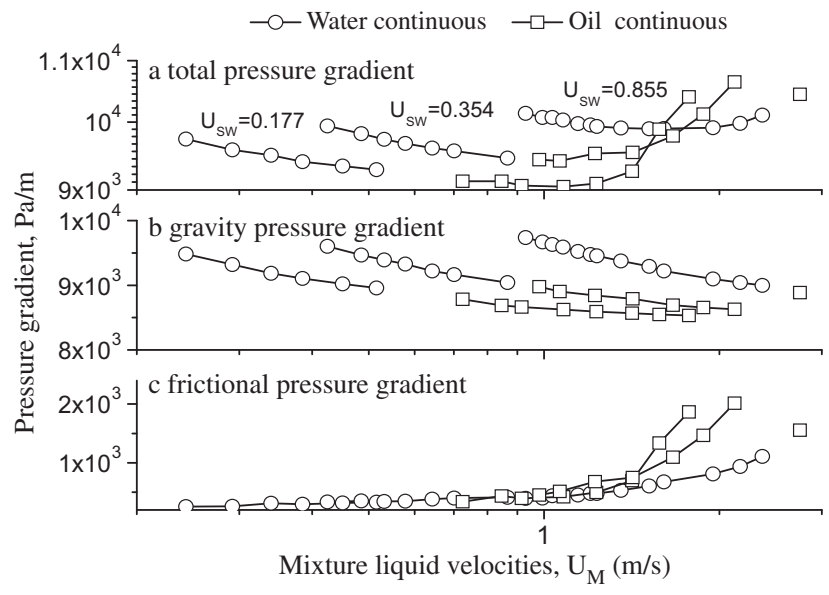

Fig. 3. Total, gravity and frictional pressure gradient for oil-water flow without gas injection as a function of mixture liquid velocities: (a) total pressure gradient, (b) gravity pressure gradient, and (c) frictional pressure gradient. low superficial water velocity. In vertical pipe flow the total pressure gradient is dominated by the gravitational part, but as the mixture liquids velocity increases the frictional component of the pressure gradient becomes more important. In Fig. 3 also the frictional pressure gradient for water continuous is lower apparently than that for oil continuous when having the same mixture velocity.

\section{Oil-water flows with gas injection}

As mentioned above the aim of this study is to investigate the influence of gas injection on flow characteristics of an oil-water flow in vertical pipe. For this purpose the three-phase flow experiments are conducted in several test series with each consisting of several test runs. Prior to the gas injection, the flow pattern of oilwater flow is shown in Fig. 2 using the scattered data points. It can be seen that the oil-water flow pattern without the gas injection is dispersed flow in the vertical test section. During the experiments, the mixture velocity of three-phase is low (Um $<3 \mathrm{~m} / \mathrm{s}$ ) and oil phase viscosity high ( $44 \mathrm{mPa}$ s) so that only bubbly flow and intermittent flow occur. This can be checked with the video recordings. By the video recording two flow patterns are identified during the experimental data: water-based dispersed flow and oil-based dispersed flow.

For a fully developed three-phase dispersed flow in a vertical pipe and neglecting the acceleration gradient, the total pressure gradient comprising of gravity pressure gradient and frictional pressure gradient can be calculated as follows:

$\left(\frac{d p}{d l}\right)_{\text {three }}=\left(\frac{d p}{d l}\right)_{g \text {-three }}+\left(\frac{d p}{d l}\right)_{f \text {-three }}$

$\left(\frac{d p}{d l}\right)_{g \text {-three }}=\left(1-\alpha_{g}\right) \rho_{m} g$

$\left(\frac{d p}{d l}\right)_{f \text {-three }}=F_{l}+F_{g l}$

here, $\rho_{m}, g$ and $\alpha_{g}$ denote, respectively, mixture density of oil-water two-phase, gravitational acceleration and the in situ gas fraction. The first term on the right hand side of Eq. (3) accounts for the frictional pressure gradient of mixture liquids flow independent of the bubbles $\left(F_{l}\right)$, and the second term describes the gas-liquid (oil and water) frictional pressure gradient due to the bubble agitation $\left(F_{g l}\right)$.

\subsection{Average in situ gas fraction}

Gas injection can change the in situ phase fraction and frictional pressure gradient of oil and water flowing in pipes (Nädler and Mewes, 1995; Xu et al., 2009). When phase inversion occurs, those changes during phase inversion can become larger. Fig. 4 shows the changes of the in situ gas fraction at fixed input gas fractions, but with a varying input oil/total liquid ratio $\left(\beta_{o}\right)$. As will be seen, the data of the in situ gas fraction reach to its lowest value around the phase inversion point. This is in agreement with previous findings in horizontal flow (Hewitt, 2005). Based on the experimental data of Descamps et al. (2007), in the region of $\beta_{o}$ around the phase inversion point the bubble velocity in the mixture liquids are larger than those away from the point. Therefore, according to the fixed input gas fraction, the slip velocity between gas and liquids will be increased gradually up to the phase inversion region and then decreases. It seems that phase inversion is still taking place at the same oil volume fraction. Based on the present experimental results, the vicinity of the phase inversion can be reflected by using the in situ gas fraction for three-phase flow under the condition of fixed input gas fraction and mixture liquid velocity. Due to the lack 


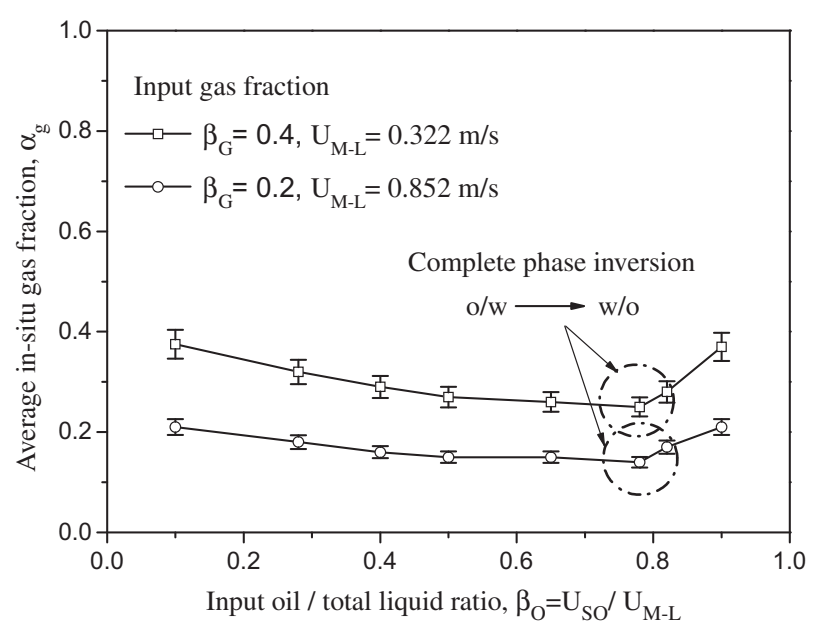

Fig. 4. Average in situ gas fraction against input oil/total liquid ratio at fixed input gas fractions for three-phase oil-gas-water vertical flow: Input gas fraction of 0.2 (circles) and 0.4 (squares).

of data reported in the literature, further detailed experiments are needed to study this phenomenon.

\subsection{Frictional pressure gradient}

The effect of the presence of gas on frictional pressure gradient in a pre-established two-phase oil-water flow is investigated. Fig. 5 shows the frictional pressure gradient for two- and threephase flow at different superficial water velocities for constant superficial gas velocity. Data are showed as a function of input oil/total liquid ratio $\left(\beta_{0}\right)$ and for three different water superficial velocities $\left(U_{\mathrm{SW}}=0.177 \mathrm{~m} / \mathrm{s}, 0.354 \mathrm{~m} / \mathrm{s}, 0.531 \mathrm{~m} / \mathrm{s}\right)$. It can be seen in Fig. 5 that the frictional pressure gradient increases with increasing $\beta_{0}$. It also increases with increasing water superficial velocity for constant $\beta_{0}$, which indicates that the frictional pressure gradient actually depends on the mixture velocity. Interestingly, it can be found in the figure that there is a similar tendency of change, for the frictional pressure gradient in two- and three-phase flows with increasing $\beta_{0}$, when superficial gas and water velocities are fixed. In general, the injection of gas phase presents the strongest effect on the frictional pressure gradient due to its velocity value.

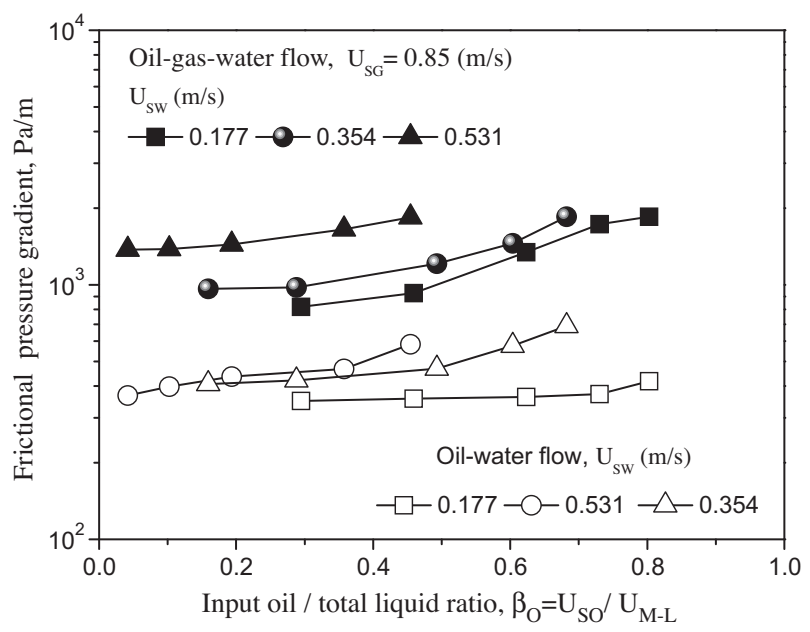

Fig. 5. Frictional pressure gradient as a function of input oil/total liquid ratio for two- and three-phase flow at three different superficial water velocities. Open symbols: oil-water flow, closed symbols: oil-gas-water flow.

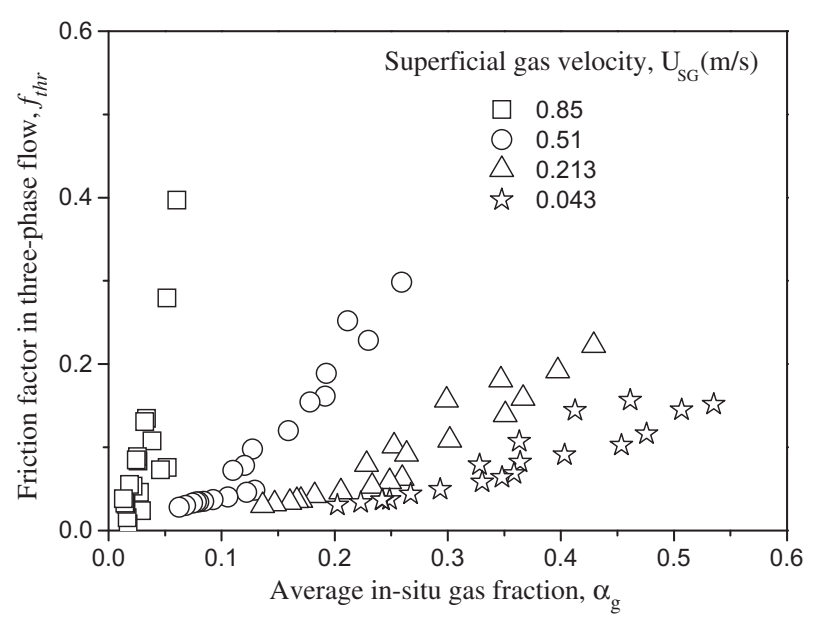

Fig. 6. Measured friction factors in three-phase flow as a function of the in situ gas fraction for four different values of superficial gas velocity.

Fig. 6 presents the measured friction factors in three-phase flow as a function of the in situ gas fraction for four different values of superficial gas velocity. As can be seen the friction factor for the three-phase flow is quickly increasing with superficial gas velocity up to the value of $0.85 \mathrm{~m} / \mathrm{s}$, at which point the mixture flow show an intermittent flow pattern and a very strong increase of the friction factor occurs (Piela et al., 2009). This means that the injection of gas into an oil-water flow increases dramatically the friction term of the pressure gradient in the Eq.(1), which is usually not dominant in vertical flow.

\subsection{Total pressure gradient}

Fig. 7 describes the influence of gas injection on the total pressure gradient for an oil-water flow at fixed mixture liquids velocity and superficial water velocity, respectively. Three-phase pressure gradient is always higher than that of two-phase flow at a low range of input oil/total liquid ratio $\left(\beta_{o}<0.7\right)$, which indicates that the presence of gas decreases considerably the gravity pressure gradient, hence the pressure gradient reduces. However, when $\beta_{o}$ is close to the phase inversion point, the injection of gas phase increases the friction factor so that the frictional term overcomes the gravity term. During this region the contribution of two terms to the total pressure gradient becomes more equal. Therefore, the injection gas in oil well should fail to decrease the pressure gradient when the in situ oil fraction remains around the phase inversion region.

A more detailed illustration for the influence of the in situ oil fraction on total pressure gradient is presented in Fig. 8. The figure presents the normalized value of gravity pressure gradient with gas injection against superficial gas velocity at three different input oil/total liquid ratios. As approximate analysis, $\mathrm{Fl}$ can be neglected base on the Fig. 5. Thus, the reduced values of gravity pressure gradient are normalized by using three-phase frictional pressure gradient as:

$\varphi_{\Delta}=\frac{(d p / d l)_{g \text {-two }}-(d p / d l)_{g \text {-three }}}{(d p / d l)_{f \text {-three }}}$

here, $\varphi_{\Delta}<1$ suggests that the gas-lift technology shows a counterproductive result. It can be found in the Fig. 8 that for $\beta_{o}$ away from the phase inversion region $\left(\beta_{o}=0.28\right), \varphi_{\Delta}$ decreases as the superficial gas velocity is increased, and but over all range of superficial gas velocity $\beta_{o}$ is large than the unity. For the values of $\beta_{o}$ around the phase inversion point $\left(\beta_{o}=0.74,0.82\right)$, the normalized pressure gradient is low than the unity when superficial gas velocity remains at a low value. 

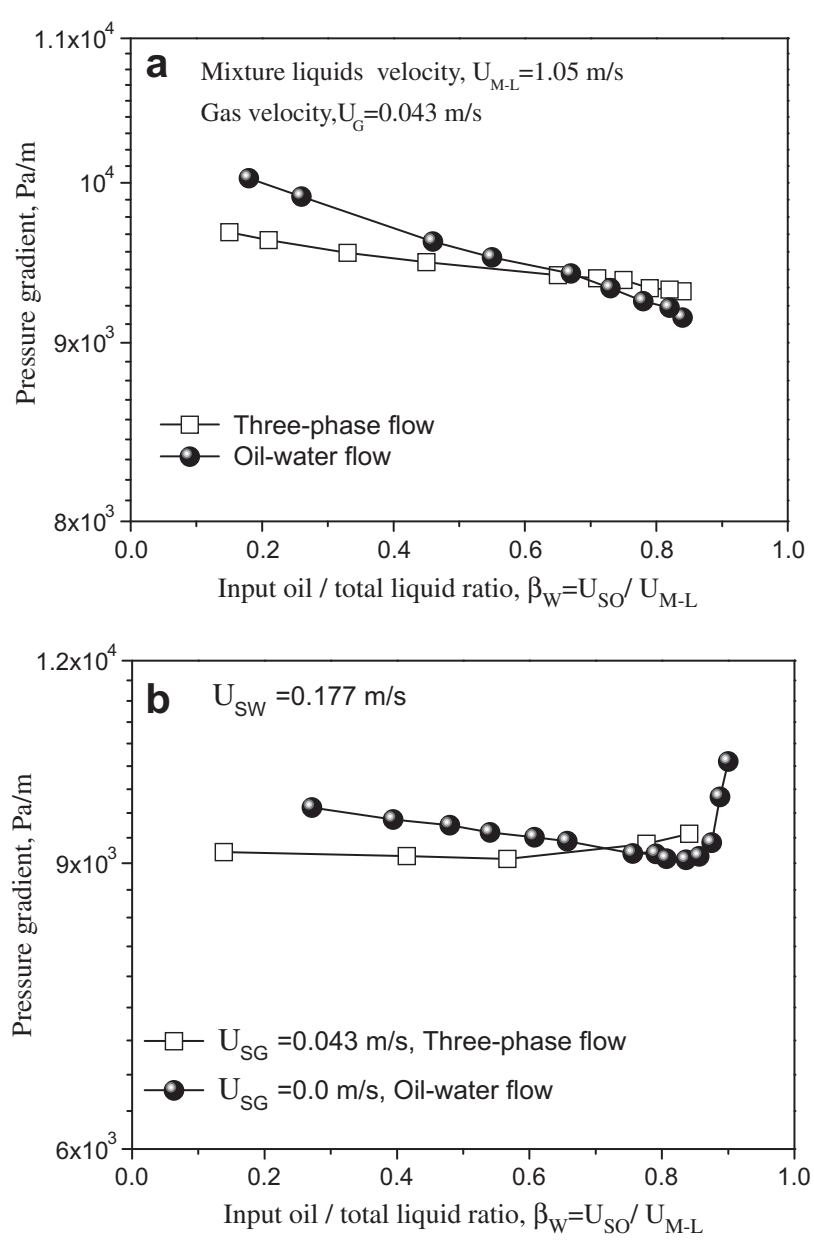

Fig. 7. Influence of gas injection on the total pressure gradient for an oil-water flow at phase inversion point: (a) at a fixed mixture liquid velocity, and (b) at a fixed superficial water velocity.

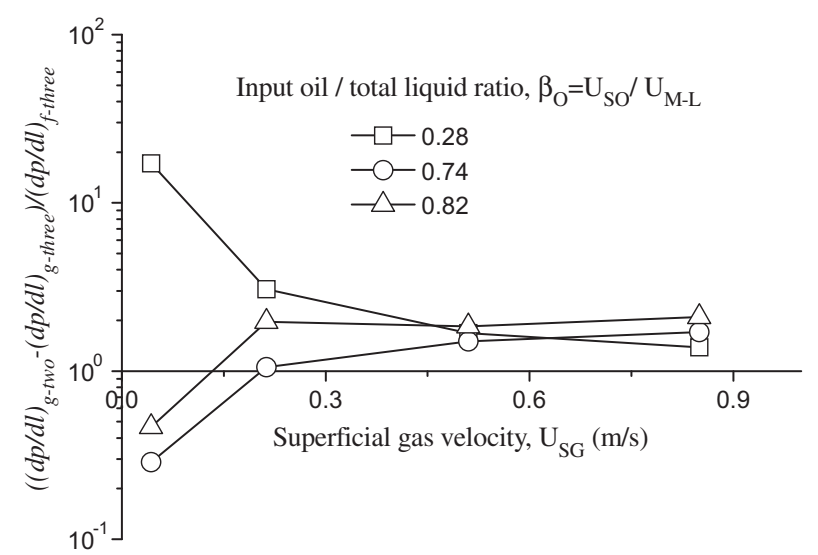

Fig. 8. The reduced value of gravity pressure gradient with gas injection normalized by using the three-phase frictional pressure gradient vs. superficial gas velocity at three different input oil/total liquid ratios.

Fig. 9 depicts the effect of the superficial velocity on the total pressure gradient in an oil-gas-water three-phase vertical flow. As can be seen in Fig. 9a, the total pressure gradient increases dramatically at high superficial gas velocity and then becomes slow at low superficial gas velocity. With the increase of superficial oil velocity, the total pressure gradient approaches the pressure
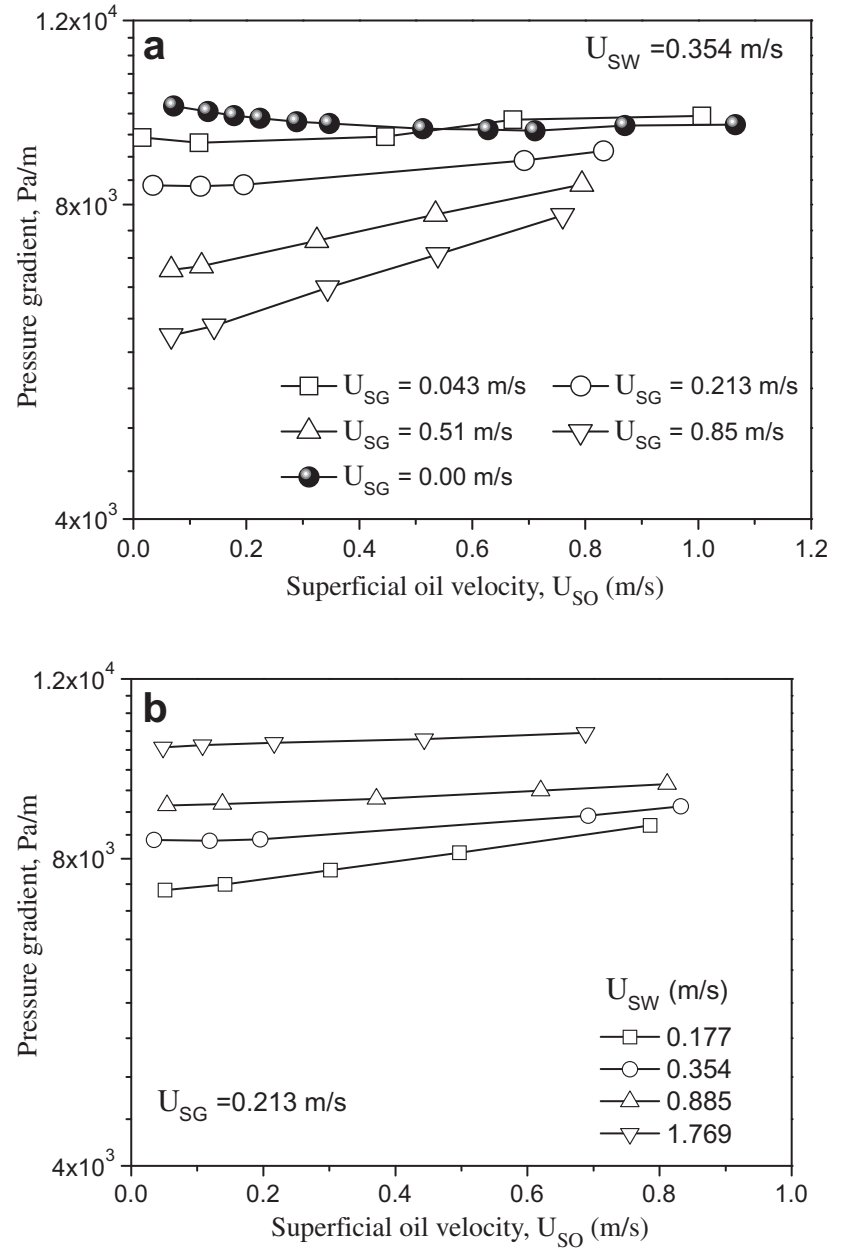

Fig. 9. Total pressure gradient in an oil-gas-water flow for oil-in-water dispersion: effect of the superficial velocity: (a) at the constant gas velocity, and (b) at the constant water velocity.

gradient of an oil-water two-phase flow. Furthermore, at a pair of high superficial gas and water velocities, the increase of superficial oil velocity show a minor impact on the pressure gradient because of the density of oil phase close to the that of water, as shown in Fig. 9b.

\subsection{Prediction of the pressure gradient for vertical bubbly flows}

There is very little information on methods for accurate calculation of pressure gradient in three-phase flow due to its complexity. In most cases, available methods for three-phase homogeneous flow have to be used, with suitable modifications for the physical properties of the combined liquid phases.

For a homogeneous flow, one method is that oil-gas-water three phases are assumed as a single phase flow. Fig. 10 shows the comparison of the measured and the predicted friction factors for the data in this work using this method. Predictions are poor. The figure exhibit a deterioration in prediction as the friction factor increased. Generally, the model of the single phase flow far underestimates the frictional pressure gradient.

Another method is that an oil-water flow is approximated as the single phase flow, the total frictional term of Eq.(3) can be expressed as a gas-liquid two-phase flow:

$\left(\frac{d p}{d l}\right)_{f \text {-three }}=\frac{4 \tau_{w}}{D}$ 


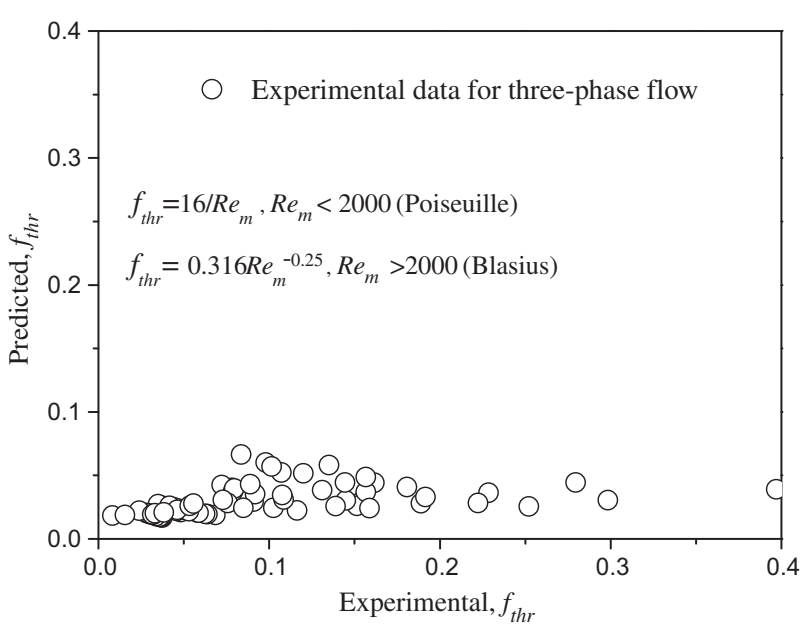

Fig. 10. Comparison of the measured and the predicted friction factors for the data in this work using the method of three-phase homogeneous flow as a single phase flow.

here $\tau_{w}$ is the shear stress, which accounts for both the friction from the liquids and the bubble induced friction.

The frictional pressure gradient of liquids (oil and water) is defined by

$\left(\frac{d p}{d l}\right)_{f}=2 f_{t w o} \rho_{m} \frac{U_{M-L}^{2}}{D}$

here $U_{M-L}=U_{S O}+U_{S W}, U_{S W}, U_{S O}$ and $f_{t w o}$ denote, respectively, the mixture liquid velocity, superficial water velocity, superficial oil velocity and oil-water mixture friction factor. In this study, Brinkman's correlation (1952) is used to calculate the effective mixture viscosity of oil and water two-phase based on the viscosities of the continuous phase and the dispersed phase and the in situ phase fraction, provided the oil and water are fully mixed:

$\mu_{e}=\mu_{c} \cdot\left(1-\alpha_{d}\right)^{-2.5}$

here $\mu$ is viscosity and $\alpha_{d}$ is the dispersion phase fraction. The subscript $e, d$ and $c$ refer to the effective mixture viscosity, dispersion phase and continuous phase, respectively.

In the past decades, many methods have been developed to predict the shear stress $\left(\tau_{w}\right)$ based on the experimental data and the physical mechanism (see a review by Guet and Ooms, 2006). Descamps et al. (2008) investigated the influence of air bubbles in liquid on the wall shear stress for the case of a vertical bubbly flow. Based on the model of Marie (1987), they suggested the law for wall shear stress ratio could be approximated as:

$\frac{\tau_{w}}{\tau_{w 0}} \approx M\left(\alpha_{g}, U_{M-L}\right)=1+\frac{10}{3\left(1-\alpha_{g}\right)} \sqrt{1.1 \alpha_{g}} \frac{0.25}{U}$

here, $U$ is the mixture velocity of gas-liquid. $\tau_{w 0}$, the wall shear stress of liquid, expended to the mixture liquids can be obtained by:

$\tau_{w 0}=\frac{f_{t w o} \cdot \rho_{t w o} \cdot U_{M-L}^{2}}{8}$

Foe a gas-liquid upward bubbly flow, Herringe and Davis (1978) also gave the ratio of the gas-liquid friction factor, with allowance for phase distribution effects, to the liquid phase friction factor as a function of void fraction (Tang and Heindel, 2006)). Provided the mixture of oil and water as a single liquid phase, the relationship is expanded in three-phase flow as:

$\frac{f_{\text {three }}}{f_{\text {two }}}=\left(1+0.22 \alpha_{g}+0.82 \alpha_{g}^{2}\right)$

then, the shear stress ratio can be expressed as:

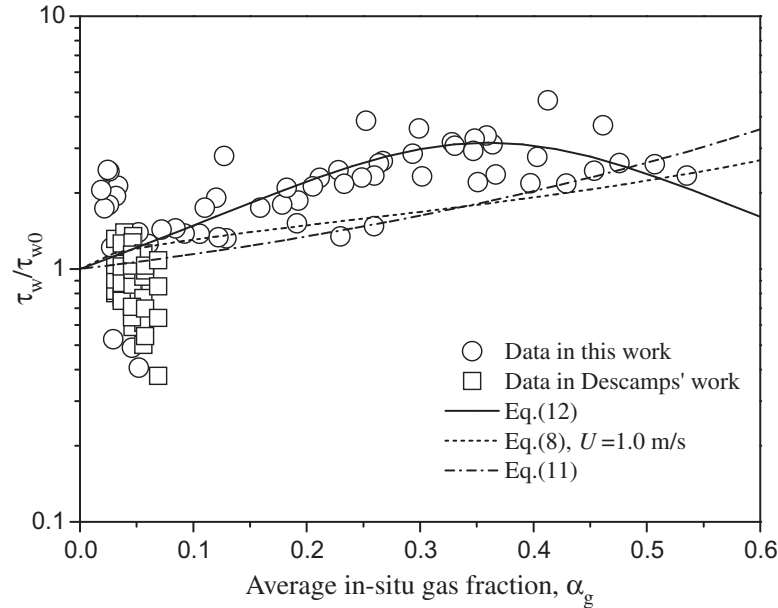

Fig. 11. Relation of the measured wall shear stress ratio and the average in situ gas fraction by using three different methods.

$\frac{\tau_{w}}{\tau_{w 0}} \approx \frac{\left(1+0.22 \alpha_{g}+0.82 \alpha_{g}^{2}\right)}{1-\alpha_{g}}$

Fig. 11 describes the measured wall shear stress ratio as an average in situ gas fraction. It is clear that as the average in situ gas fraction increases, the measured wall shear stress ratio increases up to a relatively high value, and after that it decreases. The high in situ gas fractions are observed to give internal slugs of gas in the flow. The change in the ratio with the in situ gas fraction roughly follows the line, which can be extracted from the data of the present experimental and Descamps et al.'s study, by using the least-squares analysis $\left(\alpha_{\mathrm{g}}<0.6\right.$ in this work):

$\frac{\tau_{w}}{\tau_{w 0}}=\frac{1}{1-3.8 \alpha_{g}+5.28 \alpha_{g}^{2}}$

Fig. 12 displays the comparison of the predicted frictional pressure gradient with experimental data of the present work and those reported in the literature, by using three different equations to calculate the shear stress in Eq. (5). The predictions at low gas velocity show a similar performance for three Eqs. (8), (11), and (12) but under estimation of pressure drop as the gas velocity is increased by using the Eqs. (8) and (11).

Finally, the Eq. (12) for calculating the shear stress ratio has been checked by plotting the experimental values of the total pressure gradients for three-phase flow experiments in this work and those in the literature vs. the predicted ones calculated. As Fig. 13 shows, the method gives a reasonable performance with $90 \%$ of the data falling with the $\pm 10 \%$ range. Considering that a more accurate prediction of the pressure gradient in the threephase flow is greatly complicated and difficult, the method suggested in this work is good and sufficient enough to be practically applied in industry, especially for the gas-lift technology.

\section{Conclusions}

In order to investigate the influence of gas injection on oil-water two-phase flow in vertical pipe, the characteristics of average in situ phase fraction and pressure gradient are studied experimentally. The research is restricted to three-phase bubbly or intermittent flow with a continuous liquid (oil or water).

From the experiments with gas injection, it is found that gas injection has little impact on the phase invasion. The phase inversion is still taking place at the same oil volume fraction, and the average in situ gas fraction reaches to its lowest value around 


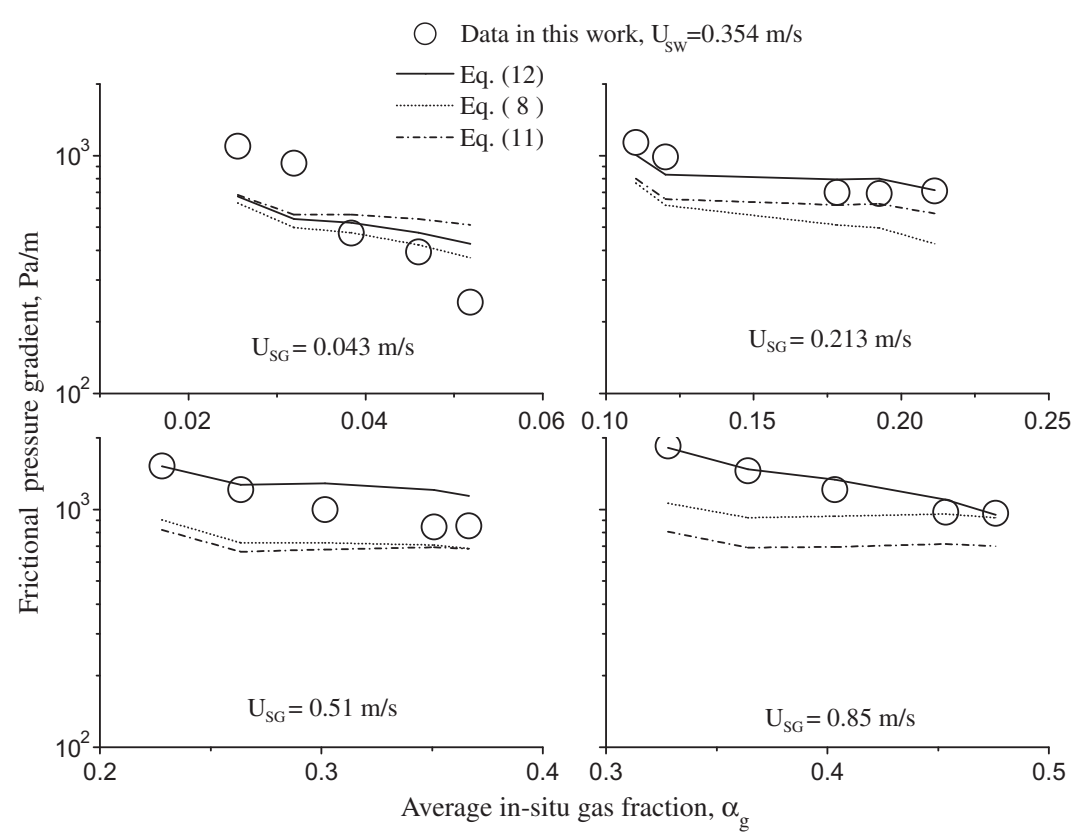

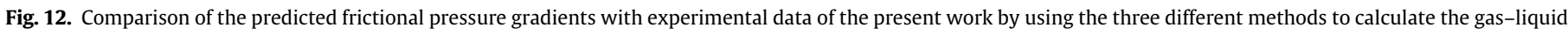

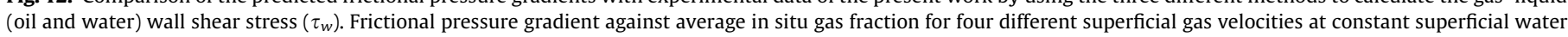
velocity, respectively.

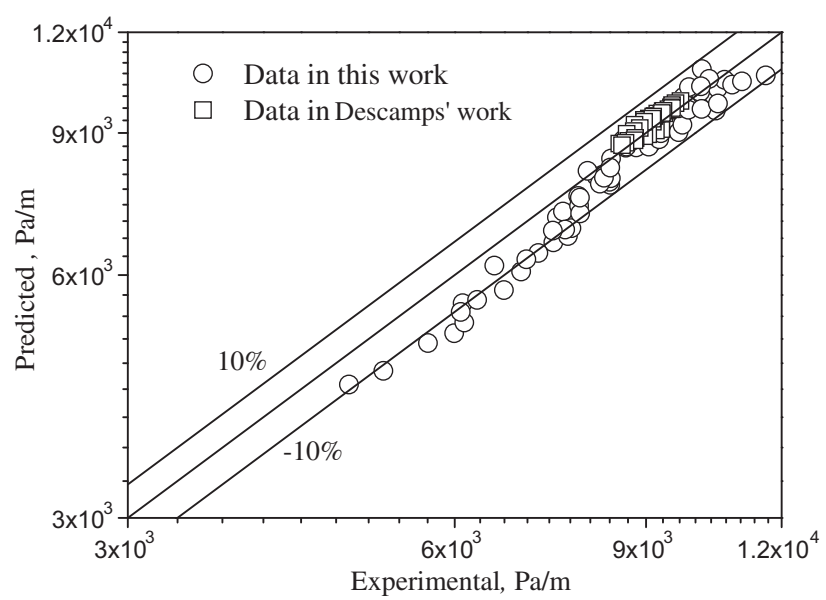

Fig. 13. Comparison between measured and predicted total pressure gradients for three-phase flow experiments in this work and those in the literature by using Eq.(12) to calculate the gas-liquid (oil and water) wall shear stress $\left(\tau_{w}\right)$.

the phase inversion point. Thus, the vicinity of the phase inversion can be reflected by using the average in situ gas fraction for threephase flow under the conditions of fixed input gas fraction and mixture liquids velocity. In comparison to oil-water flow, the presence of gas increases the mixture velocity and the frictional pressure increases. However, for the total pressure gradient, the presence of gas decreases considerably the gravity pressure gradient so that the pressure reduces. When the input oil fraction is close to the phase inversion point, the contribution of frictional and gravity terms to the total pressure gradient becomes more equal. Therefore, the injection gas in oil well should fail to decrease the pressure gradient when the in situ oil fraction remains around the phase inversion region.

Furthermore, several methods based on an oil-water flow as a single liquid flow are evaluated by using the present experimental data and a new simple method has been developed to predict the pressure gradient of three-phase vertical bubbly flow. A good agreement is obtained between theory and data in this work and those in the literature.

\section{Acknowledgments}

The authors gratefully acknowledge that the work described here was financially supported by both the National Natural Science Foundation of China (No. 10902114) and the second task in the subsea high efficiency oil/gas/water separating and metering device, the special development of national key scientific instruments in China (2011YQ120048-02).

\section{References}

Bannwart, A.C., Rodriguez, O.M.H., Trevisan, F.E., Vieira, F.F., de Carvalho, C.H.M., 2009. Experimental investigation on liquid-liquid-gas flow: flow patterns and pressure-gradient. J. Petrol. Sci. Eng. 65, 1-13.

Brinkman, H.C., 1952. The viscosity of concentrated suspensions and solutions. J. Chem. Phys. 20, 571

Cazarez, O., Montoya, D., Vital, A.G., Bannwart, A.C., 2010. Modeling of three-phase heavy oil-water-gas bubbly flow in upward vertical pipes. Int. J. Multiphase Flow 36, 439-448.

Descamps, M., Oliemans, R., Ooms, G., Mudde, R., Kusters, R., 2006. Influence of gas injection on phase inversion in an oil-water flow through a vertical tube. Int. J. Multiphase Flow 32, 311-322.

Descamps, M., Oliemans, R., Ooms, G., Mudde, R., 2007. Experimental investigation of three-phase flow in a vertical pipe: local characteristics of the gas phase for gas-lift conditions. Int. J. Multiphase Flow 33, 1205-1221.

Descamps, M.N., Oliemans, R.V.A., Ooms, G., Mudde, R.F., 2008. Air-water flow in a vertical pipe: experimental study of air bubbles in the vicinity of the wall. Exp. Fluids 45, 357-370.

Guet, S., Ooms, G., 2006. Fluid mechanical aspects of the gas-lift technique. Ann. Rev. Fluid Mech. 38, 225-249.

Herringe, R.A., Davis, M.R., 1978. Flow structure and distribution effects in gasliquid mixture flows. Int. J. Multiphase Flow 4, 461-486.

Hewitt, G.F., 2005. Three-phase gas-liquid-liquid flows in the steady and transient states. Nucl. Eng. Des. 235, 1303-1316.

Marié, J., 1987. Modeling of the skin friction and heat transfer in turbulent twocomponent bubbly flows in pipe. Int. J. Multiphase Flow 13, 309-325.

Nädler, M., Mewes, D., 1995. The effect of gas injection on the flow of immiscible liquids in horizontal pipes. Chem. Eng. Technol. 18, 156-165.

Piela, K., Delfos, R., Ooms, G., Westerweel, J., Oliemans, R.V.A., 2009. Dispersed oilwater-gas flow through a horizontal pipe. AIChE J. 55, 1090-1102.

Spedding, P.L., Woods, G.S., Raghunathan, R.S., Watterson, J.K., 2000. Flow pattern, holdup and pressure drop in vertical and near vertical two- and three-phase upflow. Trans. IChemE 78, 404-418. 
Tang, C., Heindel, T.J., 2006. Estimating gas holdup via pressure difference measurements in a cocurrent bubble column. Int. J. Multiphase Flow 32, 850863.

Woods, G.S., Spedding, P.L., Watterson, J.K., Raghunathan, R.S., 1998. Three-phase oil/water/air vertical flow. Trans. IChemE 76, 571-584.
Xu, J.-y., Li, D.-h., Guo, J., Wu, Y.-X., 2010. Investigations of phase inversion and frictional pressure gradients in upward and downward oil-water flow in vertical pipes. Int. J. Multiphase Flow 36, 930-939.

$\mathrm{Xu}, \mathrm{J} ., \mathrm{Wu}, \mathrm{Y}$. , Chang, Y., 2009. Influence of gas injection on in-situ oil fraction of an oil-water flow in horizontal pipes. Chem. Eng. Technol. 32, 1922-1928. 